\title{
Disturbance Calculation based on Space Vector Dot Product: Applications to Compensators
}

\author{
Kelly Caroline Mingorancia de Carvalho*a) \\ Naji Rajai Nasri Ama* \\ Wilson Komatsu* \\ Fernando Ortiz Martinz* \\ Ricardo Souza Figueredo* \\ Lourenço Matakas Junior*
}

\author{
Non-member \\ Non-member \\ Non-member \\ Non-member \\ Non-member \\ Non-member
}

(Manuscript received Aug. 22, 2014, revised Dec. 24, 2014)

\begin{abstract}
The ability to individually extract different types of disturbances present in AC line currents or voltages is desirable for reference signal calculation algorithms employed in power conditioners, in order to optimize the operation and design of power converters. This paper proposes a novel algorithm based on the dot product of three-dimensional space vectors in the $a b c$ coordinate system, for real-time calculation of the instantaneous positive-, negative- and zero-sequence components for individual harmonics. Certain applications are presented, including individually compensating the fundamental reactive power, selected current harmonics and current unbalances. This method is computationally simple and requires no coordinate transformation. The proposed algorithm is validated by simulation and experiments.
\end{abstract}

Keywords: amplitude and phase estimation, phase-locked loops (PLL), power quality, space vectors

\section{Introduction}

Many strategies are employed for the real time calculation of the reference signals for disturbance active compensators. One desirable feature for the reference calculation algorithm is the ability to separate the disturbance in relevant parts, since this characteristic has impact on the choice of the converter power rating and bandwidth. It is well known that reactive power and unbalance compensation requires a high power, low frequency converter. On the other hand, harmonics compensation requires high bandwidth for the converter controller, high switching frequency and operation of the converter at lower power ratings.

Among the strategies presented in the literature, it can be noted that the Instantaneous $p q$ theory, in its original form ${ }^{(1)}$, does not require a phase-locked loop (PLL) and it is convenient to compensate a large set of disturbances. However, it is not possible to extract the disturbances individual components, unless modified versions ${ }^{(2)}$ are employed, which can result in fictitious terms without physical meanings. On the other hand, Instantaneous Conservative Power theory ${ }^{(3)}$ proposes the decomposition in a number of terms with physical meaning. However, even a reactive compensation will need a high bandwidth converter to inject current harmonics that

a) Correspondence to: K.C.M. de Carvalho. E-mail:

kellymingorancia@usp.br

* Electrical Energy and Automation Department of Polytechnic School of the University of São Paulo

São Paulo, Brazil. appear when the grid voltage is distorted. This phenomenon also happens for the pq theory.

Techniques based on the Fourier series are shown in ${ }^{(4)}$, and methods based on the $a b c / d q$ transformation are also widely used $^{(5)}$. The use of multiple $d q$ frames rotating at different speeds and directions ${ }^{(6)(7)}$ enables the extraction of the individual positive- and negative-sequence harmonics. However, the strategies presented in ${ }^{(4)-(7)}$ require coordinate transformations.

Methods based on the Fortescue Transformation ${ }^{(8)}$ usually do not allow the separation of different types of disturbances. Authors of ${ }^{(9)}$ propose a new definition of instantaneous sequence components, which cannot separate the individual harmonics. Reference ${ }^{(10)}$ proposes a method based on the inner (dot) product to obtain the instantaneous fundamental positive-sequence.

This paper proposes a novel strategy to extract the instantaneous positive-, negative- and zero-sequences for each harmonic, based on the dot product (DP) of three-dimensional $a b c$ stationary frame space vectors. Additionally, a graphical explanation based on $a b c$ and $\alpha \beta 0$ coordinate systems is presented to clarify the comprehension of this method. Three cases for the minimization of load current disturbances are simulated and experimentally tested, including the individual compensation of fundamental reactive power, individual current harmonics and current unbalances.

Compared to existing methods, the proposed algorithm has a simple and alternative interpretation, requiring no coordinate transformations. Moreover, it can also be applied to 


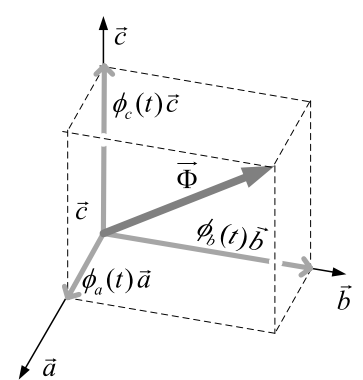

Fig. 1. Space vector in the $a b c$ system $\left(\mathfrak{R}^{3}\right)$

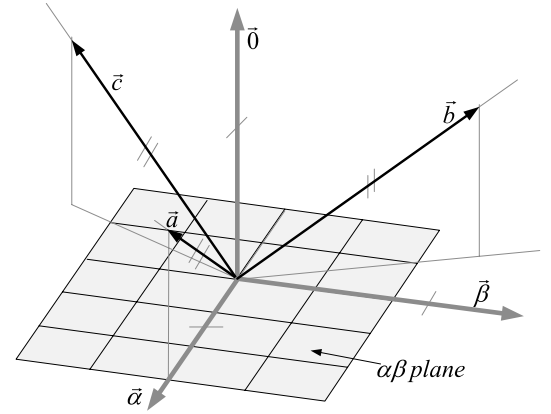

Fig. 2. $\quad a b c$ and $\alpha \beta 0$ coordinate systems $\left(\mathfrak{R}^{3}\right)$

compensate voltage disturbances such as specific harmonics, unbalances, voltage sags and voltage swells. This strategy can also be used in three-phase four-wire systems.

\section{Disturbance Calculation based on Space Vec- tor Dot Product}

Figure 1 shows an orthonormal basis $a b c$ formed by the unit length vectors $\vec{a}, \vec{b}, \vec{c}$ of $\mathfrak{R}^{3}$.

A set of time varying coordinates $\phi_{a}(t), \phi_{b}(t), \phi_{c}(t)$ defines the instantaneous space vector $\vec{\Phi}$ (Fig. 1):

$$
\vec{\Phi}=\phi_{a}(t) \vec{a}+\phi_{b}(t) \vec{b}+\phi_{c}(t) \vec{c}=\left[\phi_{a} \phi_{b} \phi_{c}\right]^{T}
$$

where $T$ is the matrix transpose operator. The coordinates $\phi_{a}$, $\phi_{b}, \phi_{c}$ can be the line to neutral voltages $v_{a}, v_{b}, v_{c}$ defining the vector $\vec{V}$, or the line currents $i_{a}, i_{b}, i_{c}$ defining the vector $\vec{I}$. The proposed algorithm uses only variables in the $a b c$ coordinate system, but its explanation is more conveniently done in the $\alpha \beta 0$ system defined in ${ }^{(11)(13)}$ and shown in Fig. 2.

Considering $\vec{\Phi}$ given by (1), the $\alpha \beta$ plane is the locus of the vectors with null instantaneous zero-sequence, defined by

$$
\phi_{a}(t)+\phi_{b}(t)+\phi_{c}(t)=0 .
$$

According to Fig. 3, an arbitrary space vector $\vec{\Phi}$ can be decomposed in a zero-sequence vector $\vec{\Phi}^{0}$ and in the vector $\vec{\Phi}_{\alpha \beta}$.

The vector $\vec{\Phi}_{\alpha \beta}$ remains on the $\alpha \beta$ plane and contains, respectively, the vectors corresponding to the $h^{\text {th }}$ order positiveand negative-sequence harmonic components $\vec{\Phi}_{h}^{+}$and $\vec{\Phi}_{h}^{-}$, as it is shown in Fig. 3. The vector $\vec{\Phi}_{h}^{+}$rotates in counterclockwise direction with $h \omega$ speed and constant amplitude $\sqrt{3 / 2} \Phi_{h}^{+}$, where $\Phi_{h}^{+}$is the peak amplitude of the $h^{\text {th }}$ order positive-sequence harmonic. The vector $\vec{\Phi}_{h}^{-}$rotates in the clockwise direction with $h \omega$ speed and constant amplitude $\sqrt{3 / 2} \Phi_{h}^{-}$, where $\Phi_{h}^{-}$is the peak amplitude of the $h^{\text {th }}$ order negative-sequence harmonic.

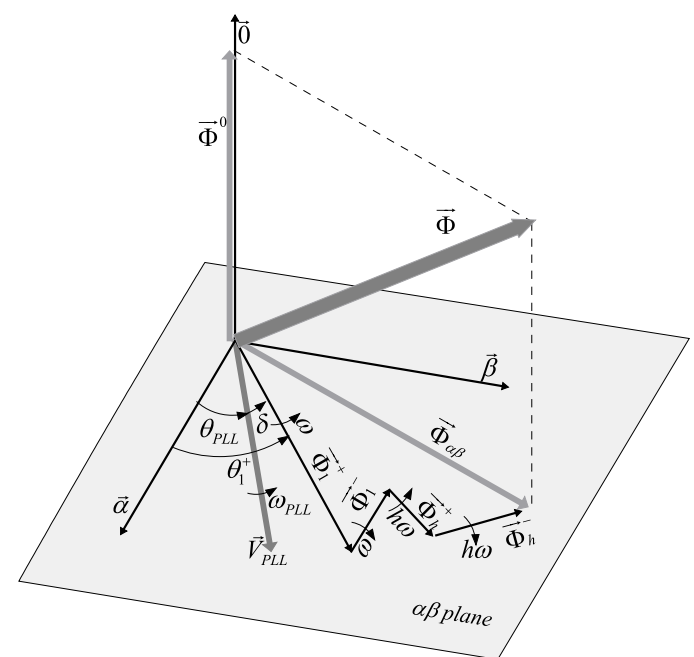

Fig. 3. Vectors $\vec{V}_{P L L}$ and $\vec{\Phi}$ (decomposed into $\vec{\Phi}_{\alpha \beta}, \vec{\Phi}_{1}^{+}$, $\left.\vec{\Phi}_{1}^{-}, \vec{\Phi}_{h}^{+}, \vec{\Phi}_{h}^{-}, \vec{\Phi}^{0}\right)$

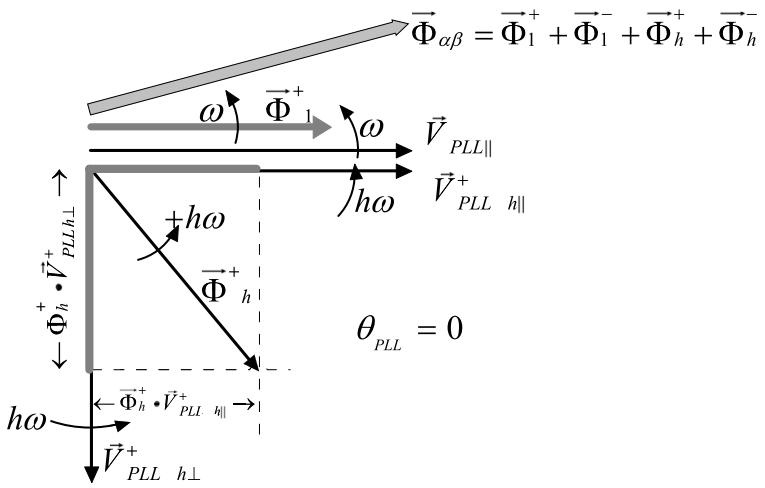

Fig. 4. Vectors $\vec{\Phi}_{1}^{+}, \vec{\Phi}_{h}^{+}, \vec{\Phi}_{\alpha \beta}, \vec{V}_{P L L \|}^{+}, \vec{V}_{P L L h \|}^{+}$and $\vec{V}_{P L L h \perp}^{+}$ in the $\alpha \beta$ plane

\subsection{Calculation of $h^{\text {th }}$ order Positive-sequence Har-} monic components $\overrightarrow{\boldsymbol{\Phi}}_{h}^{+} \quad$ The dot product of two vectors rotating at the same speed and direction is constant. Otherwise the DP will be an oscillating signal with zero mean value. This property can be used to calculate the $h^{\text {th }}$ order positive-sequence harmonic components $\vec{\Phi}_{h}^{+}=\left[\phi_{a h}^{+} \phi_{b h}^{+} \phi_{c h}^{+}\right]^{T}$. Thus, a set of orthogonal vectors $\vec{V}_{P L L h \perp}^{+}$and $\vec{V}_{P L L h \|}^{+}$, described by (3) and presented in Fig. 4 on the $\alpha \beta$ plane, which rotate in the counterclockwise direction with speed $h \omega$, and whose coordinates have unitary amplitude is necessary to calculate $\vec{\Phi}_{h}^{+}$.

$$
\left[\begin{array}{l}
\vec{V}_{P L L h \perp}^{+}=\left[\begin{array}{l}
v_{P L L a h \perp} \\
v_{P L L b h \perp} \\
v_{P L L c h \perp}
\end{array}\right]=\left[\begin{array}{l}
\sin \left(h \theta_{P L L}\right) \\
\sin \left(h \theta_{P L L}-2 \pi / 3\right) \\
\sin \left(h \theta_{P L L}+2 \pi / 3\right)
\end{array}\right] \\
\vec{V}_{P L L h \|}^{+}=\left[\begin{array}{l}
v_{P L L a h \|} \\
v_{P L L b h \|} \\
v_{P L L c h \|}
\end{array}\right]=\left[\begin{array}{l}
\cos \left(h \theta_{P L L}\right) \\
\cos \left(h \theta_{P L L}-2 \pi / 3\right) \\
\cos \left(h \theta_{P L L}+2 \pi / 3\right)
\end{array}\right]
\end{array}\right.
$$

For $h=1$, the vectors $\vec{V}_{P L L h \perp}^{+}$and $\vec{V}_{P L L h \|}^{+}$are synchronized to the positive-sequence fundamental frequency component $\vec{V}_{1}^{+}$of the mains vector $\vec{V}$. This is done by obtaining the angle $\theta_{P L L}$, which is generated by a three-phase $\mathrm{PLL}^{\dagger}$ that forces the vector $\vec{V}_{P L L 1 \|}^{+}$, calculated by imposing $h=1$ in (3),

\footnotetext{
${ }^{\dagger}$ Any three phase PLL that tracks the fundamental positive sequence of
} 


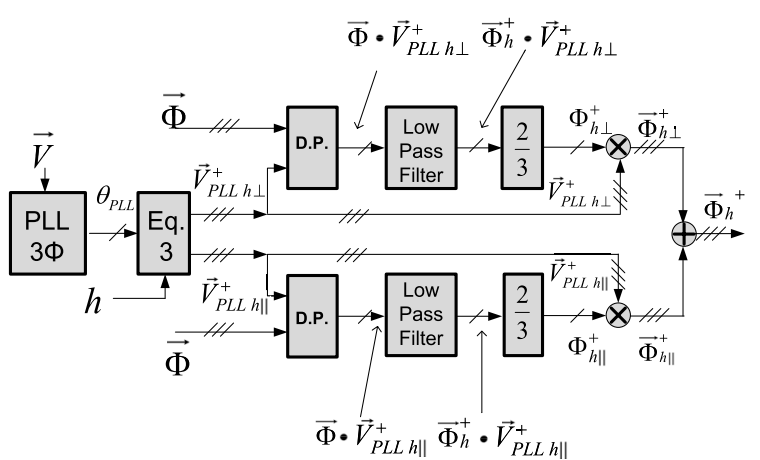

Fig. 5. Positive-sequence $\left(\vec{\Phi}_{h}^{+}\right)$extraction algorithmsingle-wire block diagram

to be instantaneously parallel to the positive-sequence vector of the mains fundamental frequency $\left(\vec{V}_{1}^{+}\right)$. The PLL strategy used in this paper is shown in Appendix.

The desired vector $\vec{\Phi}_{h}^{+}$can be decomposed in two parts, that are parallel to $\vec{V}_{P L L h \|}^{+}$and $\vec{V}_{P L L h \perp}^{+}$(Fig. 4), according to

$$
\vec{\Phi}_{h}^{+}=\left[\phi_{a h}^{+} \phi_{b h}^{+} \phi_{c h}^{+}\right]^{T}=\Phi_{h \|}^{+} \vec{V}_{P L L h \|}^{+}+\Phi_{h \perp}^{+} \vec{V}_{P L L h \perp}^{+} . \cdots
$$

If the amplitudes $\Phi_{h \|}^{+}$and $\Phi_{h \perp}^{+}$are known, $\vec{\Phi}_{h}^{+}$can be calculated by (4), as it is shown in the right side of the block diagram of Fig. 5. The evaluation of $\Phi_{h \|}^{+}$is described as follows.

The dot product $(\bullet)$ of $\vec{\Phi}$ and $\vec{V}_{P L L h \|}^{+}$is

$$
\vec{\Phi} \cdot \vec{V}_{P L L h \|}^{+}=\left(\vec{\Phi}_{1}^{+}+\vec{\Phi}_{1}^{-}+\vec{\Phi}_{h}^{+}+\vec{\Phi}_{h}^{-}+\vec{\Phi}^{0}\right) \cdot \vec{V}_{P L L h \|}^{+}
$$

Since the zero-sequence component is instantaneously orthogonal to $\vec{V}_{P L L h \perp}^{+}$and $\vec{V}_{P L L h \|}^{+}$,

$$
\vec{\Phi}^{0} \cdot \vec{V}_{P L L h \|}^{+}=0 \text {. }
$$

The other partial dot products in (5) have zero mean values, except for $\vec{\Phi}_{h}^{+} \cdot \vec{V}_{P L L h \|}^{+}$which is constant. The evaluation of the mean value of the dot product $\vec{\Phi} \cdot \vec{V}_{P L L h \|}^{+}$results in

$$
\begin{aligned}
\vec{\Phi} \cdot \vec{V}_{P L L h \|}^{+} & =\frac{1}{T_{P}} \int_{0}^{T_{P}} \vec{\Phi} \cdot \vec{V}_{P L L h \|}^{+} d t=\vec{\Phi}_{h}^{+} \cdot \vec{V}_{P L L h \|}^{+} \\
& =\sqrt{\frac{3}{2}} \Phi_{h \|}^{+} \sqrt{\frac{3}{2}}=\frac{3}{2} \Phi_{h \|}^{+}, \ldots \ldots \ldots \ldots .
\end{aligned}
$$

where $T_{P}$ is the period of the signal generated by the dot product $^{\dagger}$. Based on (7), $\Phi_{h \|}^{+}$is easily calculated by (8), by using only $a b c$ coordinates.

$$
\begin{aligned}
\Phi_{h \|}^{+} & =\frac{2}{3} \vec{\Phi} \cdot \vec{V}_{P L L h \|}^{+} \\
& =\frac{2}{3} \frac{1}{T_{P}} \int_{0}^{T_{P}}\left(\left[\phi_{a} \phi_{b} \phi_{c}\right]^{T} \cdot\left[v_{P L L a h \|} v_{P L L b h \|} v_{P L L c h \|}\right]^{T}\right) d t .
\end{aligned}
$$

Using the same reasoning, $\Phi_{h \perp}^{+}$can be evaluated by

the input signal can be used ${ }^{(10)(13)(14)}$. This paper uses the strategies implemented in the $a b c$ coordinate system (See Appendix).

$\dagger$ For usual applications the lowest frequency of the dot product occurs for unbalances, which is twice the mains frequency. This results in $T_{P}=T_{w} / 2$, where $T_{w}$ is the grid voltage period.

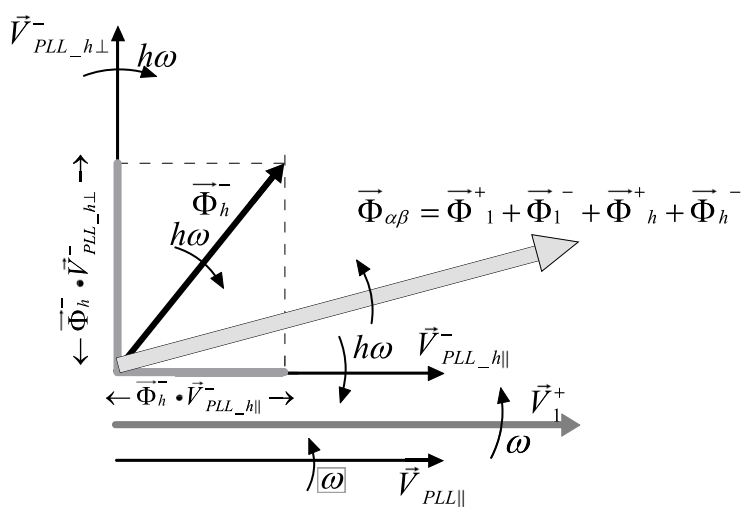

Fig. 6. Vectors $\vec{V}_{1}^{+}, \vec{\Phi}_{h}^{-}, \vec{\Phi}_{\alpha \beta}, \vec{V}_{P L L 1 \|}^{+}, \vec{V}_{P L L h \|}^{-}$and $\vec{V}_{P L L h \perp}^{-}$ in the $\alpha \beta$ plane

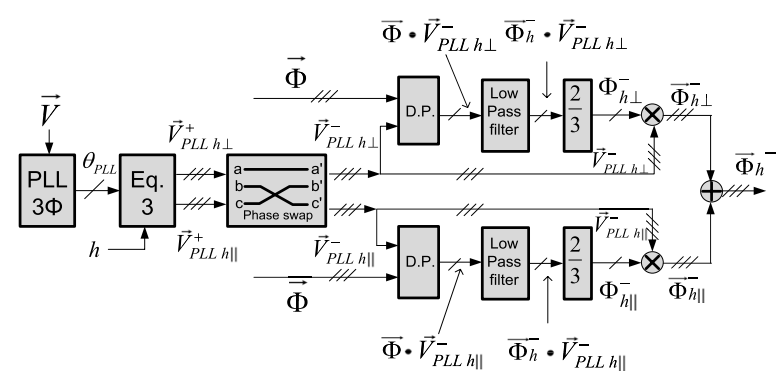

Fig. 7. Negative-sequence $\left(\vec{\Phi}_{h}^{-}\right)$extraction algorithmsingle-wire block diagram

$$
\begin{aligned}
\Phi_{h \perp}^{+} & =\frac{2}{3} \vec{\Phi} \cdot \vec{V}_{P L L h \perp}^{+} \\
& =\frac{2}{3} \frac{1}{T_{P}} \int_{0}^{T_{P}}\left(\left[\phi_{a} \phi_{b} \phi_{c}\right]^{T} \cdot\left[\begin{array}{lll}
v_{P L L h \perp} v_{P L L b h \perp} v_{P L L c h \perp}
\end{array}\right]^{T}\right) d t .
\end{aligned}
$$

The mean value can be obtained by using a low pass filter. Figure 5 presents the positive-sequence extraction algorithm in a single-wire block diagram, where $\vec{\Phi} \cdot \vec{V}_{P L L h \|}^{+}$is low pass filtered, originating $\vec{\Phi}_{h}^{+} \cdot \vec{V}_{P L L h \|}^{+}$. This signal, followed by a gain 2/3 results in the magnitude of the projection of $\vec{\Phi}_{h}^{+}$on the vector $\vec{V}_{P L L h \|}^{+}$(see Fig. 4), named as $\Phi_{h \|}^{+}$. The instantaneous values of the coordinates of $\vec{\Phi}_{h \|}^{+}$are finally obtained by calculating $\Phi_{h \|}^{+} \vec{V}_{P L L h \|}^{+}$. The same reasoning is done for the orthogonal component $\vec{\Phi} \cdot \vec{V}_{P L L h \perp}^{+}$, resulting in the extracted positive-sequence components of the $h^{\text {th }}$ harmonics, given by (4).

2.2 Calculation of $\boldsymbol{h}^{\text {th }}$ order Negative-sequence Harmonic Components $\overrightarrow{\boldsymbol{\Phi}}_{\boldsymbol{h}}^{-} \quad$ The negative-sequence harmonics $\vec{\Phi}_{h}^{-}=\left[\phi_{a h}^{-} \phi_{b h}^{-} \phi_{c h}^{-}\right]^{T}$ can be obtained by substituting the pair $\vec{V}_{P L L h \|}^{+}, \vec{V}_{P L L h \perp}^{+}$by a pair of negative-sequence orthogonal vectors $\vec{V}_{P L L h \|}^{-}$and $\vec{V}_{P L L h \perp}^{-}$, which rotates with speed $h \omega$ in the clockwise direction, as it is shown in Fig. 6. These two vectors are easily obtained by swapping the signals corresponding to phases $b$ and $c$ in (3). This is done by the "phase swap" block in the single-wire diagram of Fig. 7.

2.3 Calculation of $\boldsymbol{h}^{\text {th }}$ order Zero-sequence Harmonic Components $\overrightarrow{\boldsymbol{\Phi}}_{\boldsymbol{h}}^{\mathbf{0}} \quad$ The calculation of $\vec{\Phi}_{h}^{0}=\left[\phi_{a h}^{0} \phi_{b h}^{0} \phi_{c h}^{0}\right]^{T}$ is based on the dot products $\vec{\Phi} \cdot \vec{V}_{P L L h \|}^{0}$ and $\vec{\Phi} \cdot \vec{V}_{P L L h \perp}^{0}$, where 


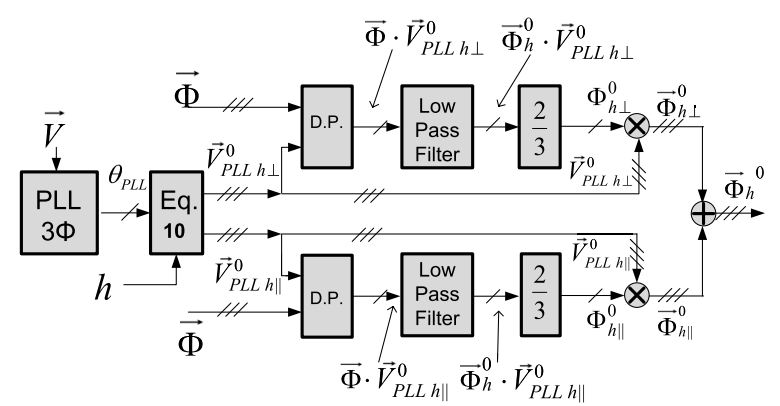

Fig. 8. Zero-sequence $\left(\vec{\Phi}_{h}^{0}\right)$ extraction algorithm-singlewire block diagram

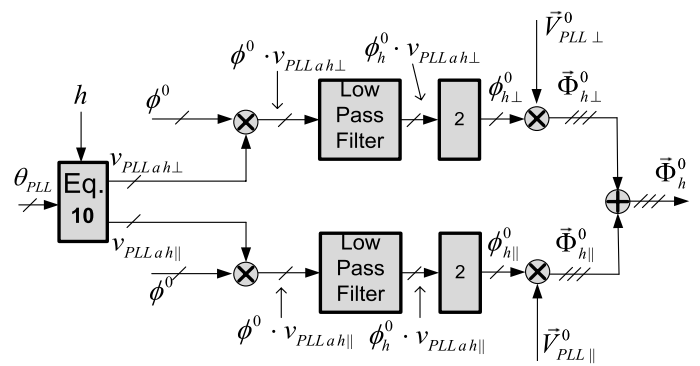

Fig. 9. Simplified zero-sequence $\left(\vec{\Phi}_{h}^{0}\right)$ extraction algorithm single-wire block diagram

$\vec{V}_{P L L h \|}^{0}$ and $\vec{V}_{P L L h \perp}^{0}$ are zero-sequence vectors with $h \omega$ frequency, given by

$$
\left[\begin{array}{l}
\vec{V}_{P L L h \|}^{0}=v_{P L L a h \|}\left[\begin{array}{lll}
1 & 1 & 1
\end{array}\right]^{T}=\cos \left(h\left(\theta_{P L L}\right)\right)\left[\begin{array}{lll}
1 & 1 & 1
\end{array}\right]^{T} \\
\vec{V}_{P L L h \perp}^{0}=v_{P L L a h \perp}\left[\begin{array}{lll}
1 & 1 & 1
\end{array}\right]^{T}=\cos \left(h\left(\theta_{P L L}+\pi / 2\right)\right)\left[\begin{array}{lll}
1 & 1 & 1
\end{array}\right]^{T}
\end{array}\right.
$$

In order to avoid unnecessary calculation, the block diagram in Fig. 8 can be simplified into the one shown in Fig. 9. The instantaneous phase and amplitudes of a zero-sequence component signal are equal. Therefore, the product of only one term of the vector $\vec{\Phi}^{0}=\left[\phi^{0} \phi^{0} \phi^{0}\right]^{T}$ is needed to calculate the zero- $h^{\text {th }}$ order sequence component by means of

$$
\phi^{0}=\frac{\phi_{a}+\phi_{b}+\phi_{c}}{3} \text {. }
$$

\section{Application to Parallel Disturbance Compen- sators-Simulation Results}

Figure 10 shows the grid voltages $\left(220 \mathrm{~V}_{\mathrm{RMS}}, 60 \mathrm{~Hz}\right)$, a delta connected RL switched load (Load 1: $R_{1}=33 \Omega, L_{1}=$ $400 \mathrm{mH}$ ), a non linear load composed by a three-phase diode rectifier $\left(\operatorname{Load} 2: R_{2}=370 \Omega, L_{2}=1.7 \mathrm{mH}, C_{2}=47 \mu \mathrm{F}\right)$ and a three-phase voltage source converter which compensates a set of chosen load disturbances. The converter is rated for $220 \mathrm{~V}$ line to line RMS voltage, 3.5 A RMS line current, $350 \mathrm{~V} \mathrm{DC}$-link voltage, for line inductor filters of $10 \mathrm{mH}$ and $800 \mu \mathrm{F}$ DC side capacitor.

Closing only switch $S_{1}$ of Load 1 results in a single-phase RL load connected between phases $a$ and $b$ (unbalanced load with reactive power). Simultaneous closure of $S_{1}$ and $S_{2}$ results in a balanced three-phase load with reactive power. Switch $S_{3}$ of Load 2 connects to the grid a diode rectifier circuit, with LC filter operating in discontinuous mode, providing distorted currents to the tests.

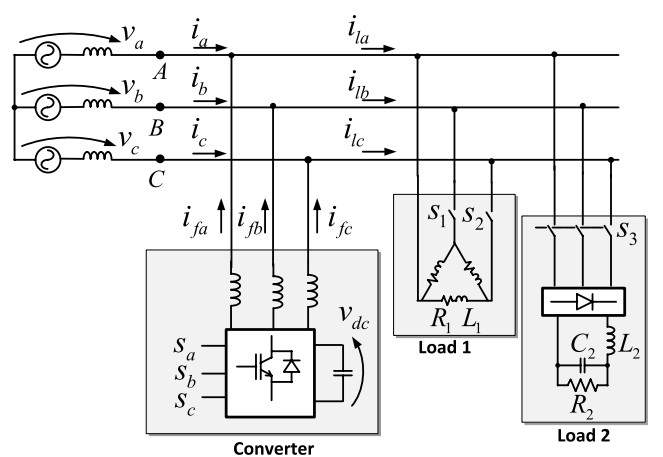

Fig. 10. Simulation and Experimental setup: VSC converter and loads

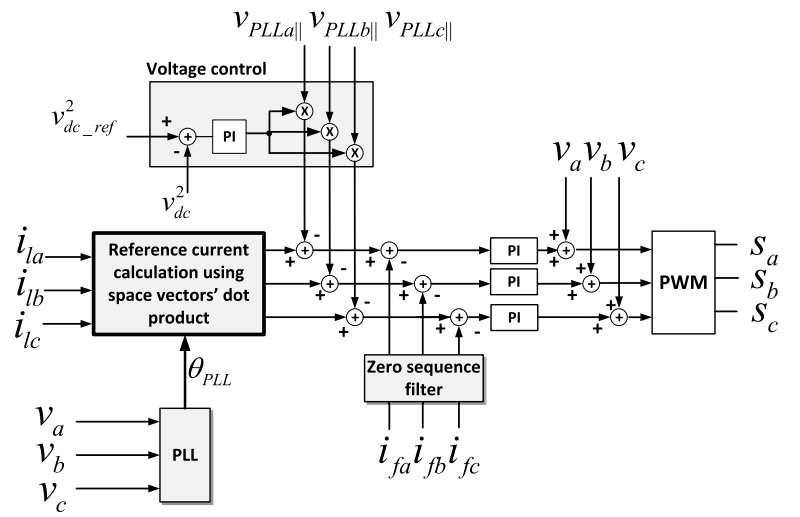

Fig. 11. Block Diagram of the control loops

Figure 11 presents the control diagram for the converter of Fig. 10, including the reference current calculator, the DC voltage control loop, the current tracking loop, the PLL and the PWM block. To maintain coherence with the proposed algorithms for disturbance calculation, all the blocks in Fig. 11 are implemented in $a b c$ system, with no coordinate transformation.

Current loop employs three digital proportional integral (PI) controllers (one for each phase), with the anti-windup algorithm presented in ${ }^{(11)}$. To avoid the well known loss of controllability of three-phase, three-wire converters using three independent controllers, this paper subtracts the instantaneous zero-sequence component, given by $\left(i_{f a}+i_{f b}+i_{f c}\right) / 3$, from the measured filter currents $i_{f a}, i_{f b}, i_{f c}$. The current loop PI controller parameters were calculated to set the damping factor of the closed loop poles to 0.4 , resulting in $K_{p}=40.4$ and $T_{i}=402 \mu \mathrm{s}$. The transfer function of the PI controller is described in (12) and its discrete form is shown in (13) using backward Euler method. Besides that, a feed forward of the grid voltage is also included, to improve the disturbance and transient response.

$$
\begin{aligned}
& G_{P I}(s)=K_{P}\left(1+\frac{1}{T_{i} s}\right) \cdots \cdots \cdots \\
& G_{P I}(z)=K_{P}\left(1+\frac{T}{T_{i}\left(1-z^{-1}\right)}\right) \cdots
\end{aligned}
$$

Furthermore, three single update sampled PWM are employed. Instantaneous zero-sequence is added to the PWM reference signal to emulate Space Vector PWM behavior ${ }^{(12)}$. Carrier and sampling frequency were set to $12 \mathrm{kHz}$ and grid frequency is $60 \mathrm{~Hz}$. 


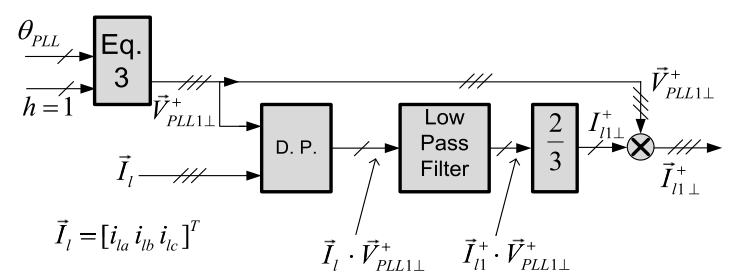

Fig. 12. Reference Calculation Block of Fig. 11 for the Fundamental Reactive Current Compensator
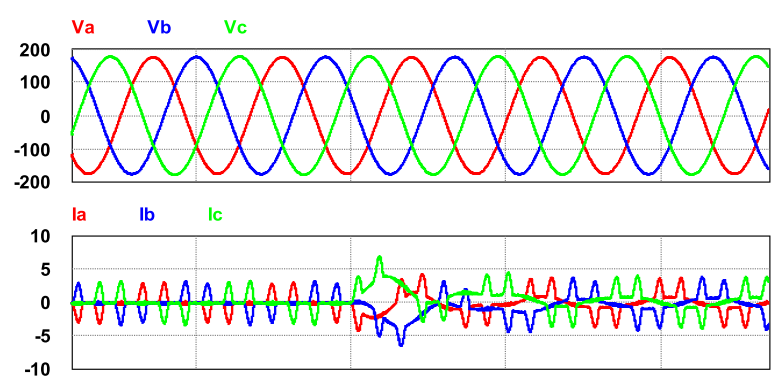

$-10$

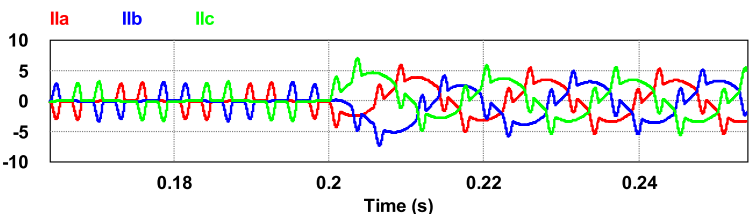

Fig. 13. Simulated results for fundamental reactive power compensator: top - line to neutral voltages $\left(v_{a}, v_{b}, v_{c}\right)$, middle: line currents $\left(i_{a}, i_{b}, i_{c}\right)$, bottom- load currents $\left(i_{l a}, i_{l b}, i_{l c}\right)$

The PLL block is based on dot product PLL ${ }^{(13)}$, also known as p-PLL, and it is presented in Appendix. The PLL PI controller gains were adjusted as described in ${ }^{(14)}$, resulting in $K_{P P L L}=139.426$ and $K_{I P L L}=0.574$. The DC voltage control loop employs a PI compensator and regulates $v_{d c}^{2}$ instead of $v_{d c}$ for exact linearization of the DC loop transfer function: The controller parameters are set to $K_{p v}=5.94 \times 10^{-5}$ and $T_{i v}$ $=49 \mathrm{~ms}$, which results in a settling time equal to 9 cycles of mains frequency.

The proposed method to calculate positive- and negativesequences of voltage and currents is applied to the three different disturbance compensators described below:

- Fundamental Reactive Currents Compensation;

- Fundamental Negative-Sequence Compensation;

- Selective Current Harmonics Compensation.

3.1 Fundamental Reactive Currents Compensation

The calculation of the reference signals for the fundamental reactive current compensator, described in the block diagram of Fig. 12, can be derived from Fig. 5 by:

- imposing the load currents as input vector $\vec{\Phi}=\vec{I}_{l}=$ $\left[\begin{array}{lll}i_{l a} & i_{l b} & i_{l c}\end{array}\right]^{T}$

- imposing $h=1$;

- using only the upper signal path corresponding to the current components orthogonal to the positive-sequence of the fundamental grid voltages.

Moving average low pass filter with a sampling frequency of $12 \mathrm{kHz}$ and a window width of $T_{w} / 2$ (where $T_{W}$ is the grid voltage period) was employed for the three compensators, resulting in a filter settling time of $T_{W} / 2$. Simulation results were obtained by using the PSIM software. Figure 13 shows line to neutral voltages, grid currents and load currents for
Table 1. Calculated values of fundamental positivesequence of grid and load currents-reactive power compensation-Simulation results

\begin{tabular}{|c|c|c|}
\hline & Grid (A - peak) & Load (A - peak) \\
\hline$t<0.2 s$ & $0.936-0.7^{\circ}$ & $0.936 \square 0.1^{\circ}$ \\
\hline$t \gg 0.2 s$ & $1.712\left\lfloor 2.0^{\circ}\right.$ & $3.711-63.4^{\circ}$ \\
\hline
\end{tabular}

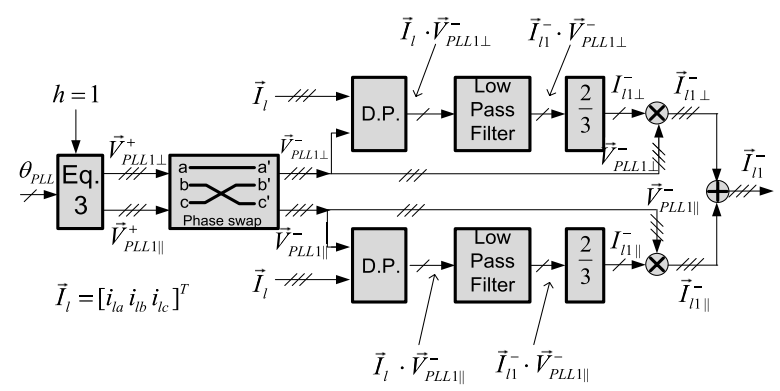

Fig. 14. Reference Calculation Block of Fig. 11 for the Fundamental Negative-Sequence Current Compensator

the fundamental reactive power compensation. Steady state operation with non linear Load $2\left(S_{3} \mathrm{On}\right)$ is presented until $0.2 \mathrm{~s}$. At $0.2 \mathrm{~s}$ switches $S_{1}$ and $S_{2}$ are turned on, including Load 1 (linear balanced RL load).

Table 1 summarizes the calculated values of the positivesequence of the load and the grid fundamental currents (peak values) for $t<0.2 \mathrm{~s}$ and $t \gg 0.2 \mathrm{~s}$. The peak value of the grid voltage positive-sequence is $179.63 \underline{0}^{\circ} \mathrm{V}$.

Simulations show that reactive fundamental currents are adequately compensated. After $0.2 \mathrm{~s}$ the current amplitude was reduced from $3.711 \mathrm{~A}$ to $1.712 \mathrm{~A}$ and the phase angle was reduced from $-63.4^{\circ}$ to $2.0^{\circ}$. Time response is expected to be equal to the moving average filter width $\left(T_{W} / 2\right)$, and thus the longer transient times in Fig. 13 are explained by the RL load large time constant ( $\tau=L_{1} / R_{1} \sim 12 \mathrm{~ms}$ ), where the steady state is reached in about 2 cycles. Moreover, current harmonics do not disturb the operation of the reactive power compensator. This is especially important for high power converters, which will be able to inject only mains frequency currents, and operate with lower switching frequencies.

3.2 Fundamental Negative-sequence Compensation

The calculation of the reference signals for the fundamental negative-sequence current compensator, described in the block diagram of Fig. 14, can be obtained from Fig. 7 by:

- imposing the load currents as input vector $\vec{\Phi}=\vec{I}_{l}=$ $\left[i_{l a} i_{l b} i_{l c}\right]^{T}$

- $\operatorname{imposing} h=1$.

Figure 15 shows line to neutral voltages, grid currents and load currents for the Fundamental Negative-Sequence Current Compensator. Steady state operation with unbalanced linear load (Load 1 with only $S_{1}$ On) is presented until $0.2 \mathrm{~s}$. At $0.2 \mathrm{~s}$ the load becomes a balanced inductive load $\left(S_{1}\right.$ and $S_{2}$ are turned on).

Table 2 presents the calculated values of the positive- and negative-sequences of the load and the grid fundamental currents (peak values) for $t<0.2 \mathrm{~s}$ and $t \gg 2 \mathrm{~s}$. The peak of the grid voltage positive-sequence is $179.63\left\lfloor 0^{\circ} \mathrm{V}\right.$.

For $t<0.2 \mathrm{~s}$ the negative-sequence is adequately compensated (reduced from 1.707 A to $0.040 \mathrm{~A}$ ). The long settling time for $t>0.2 \mathrm{~s}$ is again explained by the RL load transient. 

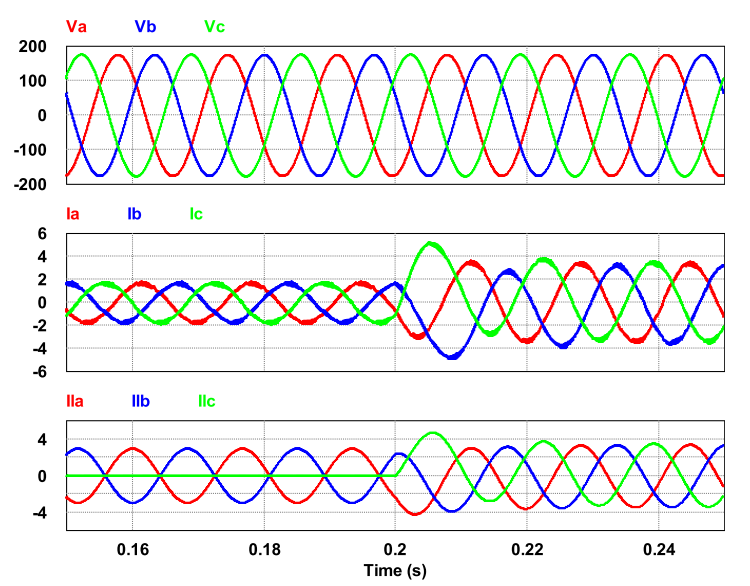

Fig. 15. Simulated results for fundamental negativesequence compensator: top - line to neutral voltages $\left(v_{a}, v_{b}, v_{c}\right)$, middle: line currents $\left(i_{a}, i_{b}, i_{c}\right)$, bottom- load currents $\left(i_{l a}, i_{l b}, i_{l c}\right)$

Table 2. Calculated values of fundamental symmetrical components for grid and load currents - negative component compensation- Simulation results

\begin{tabular}{|c|c|c|c|}
\hline & & $\mathrm{I}_{\text {grid }}$ & $\mathrm{I}_{\mathrm{load}}$ \\
\hline \multirow{2}{*}{$t<0.2 s$} & positive & $1.709-77.2^{\circ}$ & $1.707 \mid-77.7^{\circ}$ \\
\cline { 2 - 4 } & negative & $0.040-172.3^{\circ}$ & $1.707 \square-17.7^{\circ}$ \\
\hline \multirow{2}{*}{$t \gg 0.2 s$} & positive & $3.427-77.5^{\circ}$ & $3.415 \square-77.7^{\circ}$ \\
\cline { 2 - 4 } & negative & 0.000 & 0.000 \\
\hline
\end{tabular}

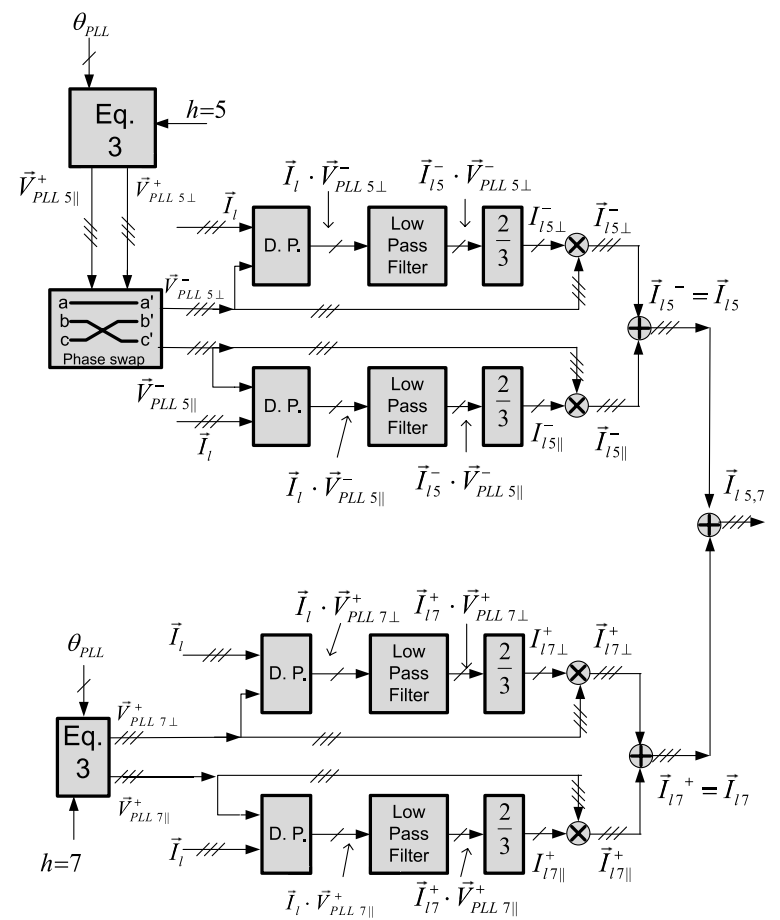

Fig. 16. Reference Calculation Block of Fig. 11 for the compensation of $5^{\text {th }}$ and $7^{\text {th }}$ harmonics

\subsection{Selective Current Harmonics Compensation}

The calculation of the reference signals for specific current harmonics compensation described in the block diagram of Fig. 16, considering only the $5^{\text {th }}$ and $7^{\text {th }}$ harmonics, can be obtained from Fig. 5 and Fig. 7 by:

- imposing the load currents as input vector $\vec{\Phi}=\vec{I}_{l}=$ $\left[\begin{array}{lll}i_{l a} & i_{l b} & i_{l c}\end{array}\right]^{T}$

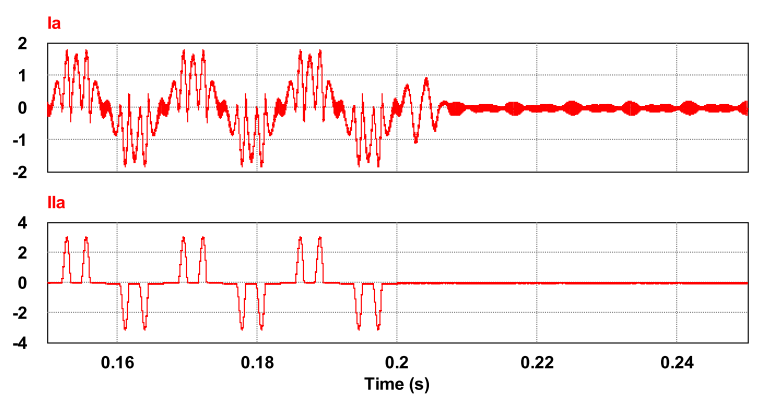

Fig. 17. Simulated results for current harmonics: top line current $\left(i_{a}\right)$, bottom- load current $\left(i_{l a}\right)$

Table 3. Calculated $1^{\text {st }}, 5^{\text {th }}$ and $7^{\text {th }}$ current components (A peak) for specific current harmonics compensationSimulation results

\begin{tabular}{|c|c|c|}
\hline & $\mathrm{I}_{\text {grid }}$ & $\mathrm{I}_{\text {load }}$ \\
\hline 1 & 0.952 & 0.947 \\
\hline 5 & 0.002 & 0.840 \\
\hline 7 & 0.003 & 0.742 \\
\hline 11 & 0.501 & 0.500 \\
\hline 13 & 0.376 & 0.376 \\
\hline
\end{tabular}

- considering that the three-phase load currents have the same waveform and are equally displaced. This results in a negative-sequence $5^{\text {th }}$ harmonic and a positive-sequence $7^{\text {th }}$ harmonic. As a consequence, Fig. 16 simultaneously uses the algorithms shown in Figs. 7 and 5, with $h=5$ and $h=7$ respectively.

Since the PI controller with sampling frequency of $12 \mathrm{kHz}$ presents excessive tracking error for the $5^{\text {th }}$ and $7^{\text {th }}$ harmonics, the harmonics compensator replaces this controller by three proportional plus resonant (PR) controllers tuned at $60 \mathrm{~Hz}, 300 \mathrm{~Hz}$ and $420 \mathrm{~Hz}$ respectively, according to (14).

$$
\begin{aligned}
G_{P R}(s)= & 40.4\left(1+\frac{(2 \cdot 2 \pi 60) s}{s^{2}+(2 \pi 60)^{2}}+\frac{0.1 \cdot(2 \cdot 5 \cdot 2 \pi 60) s}{s^{2}+(5 \cdot 2 \pi 60)^{2}}\right. \\
& \left.+\frac{0.1 \cdot(2 \cdot 7 \cdot 2 \pi 60) s}{s^{2}+(7 \cdot 2 \pi 60)^{2}}\right) . \cdots \cdots \cdots \cdots(14)
\end{aligned}
$$

Using Tustin with pre-warping ${ }^{(15)}$ method in (14) results in the following discrete transfer function.

$$
\begin{aligned}
G_{P R}(z)= & 40.4+\frac{1.269\left(1-z^{2}\right)}{1-1.999 z^{-1}+z^{-2}}+\frac{6.320\left(1-z^{-2}\right)}{1-1.975 z^{-1}+z^{-2}} \\
& +\frac{8.884\left(1-z^{-2}\right)}{1-1.952 z^{-1}+z^{-2}} \cdots \cdots \ldots \ldots \ldots \ldots(15)
\end{aligned}
$$

Figure 17 presents simulated line and load current waveforms for the compensation case of Fig. 16. For $t<0.2 \mathrm{~s} S_{3}$ is on, and the non linear load is connected to the grid. For $t>0.2 \mathrm{~s} S_{3}$ is off.

Table 3 shows calculated values of the $1^{\text {st }}, 5^{\text {th }}$ and $7^{\text {th }}$ harmonics for the grid and load phase a current, for $t<0.2 \mathrm{~s}$.

The $5^{\text {th }}$ and $7^{\text {th }}$ harmonics are adequately attenuated. Figure 17 shows that the settling time is around half of mains period, which corresponds to the employed moving average filter window size. For $t>0.2 \mathrm{~s}$ grid harmonic components presented in Table 3 are null, since there is no load connected to the grid. The distorted grid current waveform of Fig. 17 is due to the high amplitude of $11^{\text {th }}$ and $13^{\text {th }}$ components, which were not expected to be compensated. 

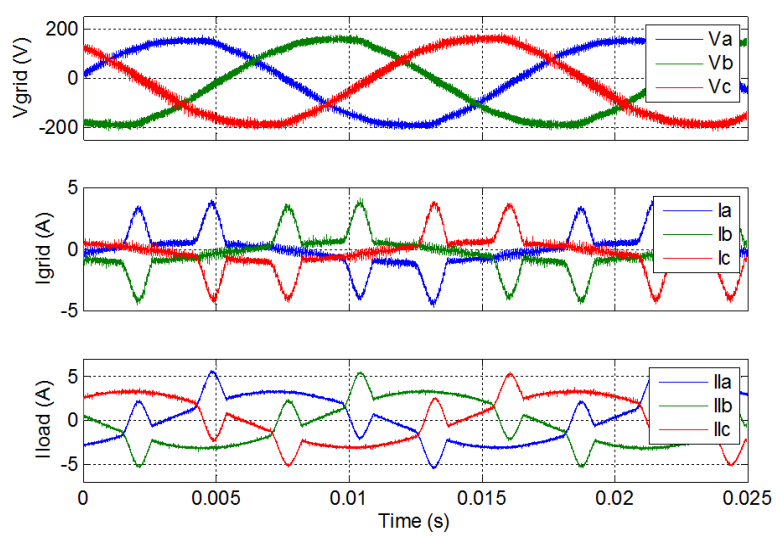

Fig. 18. Experimental results for fundamental reactive power compensator: top - line to neutral voltages $\left(v_{a}, v_{b}, v_{c}\right)$, middle: line currents $\left(i_{a}, i_{b}, i_{c}\right)$, bottom- load currents $\left(i_{l a}, i_{l b}, i_{l c}\right)$

Table 4. Calculated Symmetrical components for reactive power compensation- Experimental results

\begin{tabular}{|c|c|c|c|}
\hline & $\mathrm{V}_{\text {grid }}(\mathrm{Vpeak})$ & $\mathrm{I}_{\text {prid }}(\mathrm{Apeak})$ & $\mathrm{I}_{\text {load }}($ Apeak \\
\hline positive & $176.15-0.5^{\circ}$ & $1.62\left[-3.5^{\circ}\right.$ & $3.42\left[-62.6^{\circ}\right.$ \\
\hline negative & $0.82\left\lfloor-149.6^{\circ}\right.$ & $0.02\left\lfloor-40.8^{\circ}\right.$ & $0.04\left\lfloor-41.1^{\circ}\right.$ \\
\hline zero & $1.25-106.8^{\circ}$ & 0.00 & $0.01 \mid-62.6^{\circ}$ \\
\hline
\end{tabular}

If the complete harmonics compensation is desired, $h=1$ must be used in Fig. 5 to calculate the fundamental positivesequence and, $h=1$ must also be used in Fig. 7 to calculate the fundamental negative-sequence. The total harmonic current is calculated by $\sum \vec{I}_{h}=\vec{I}-\vec{I}_{1}^{+}-\vec{I}_{1}^{-}$.

\section{Experimental Results}

Control loops and PWM blocks of the experimental setup were implemented in a TMS320F28335 DSP. Waveforms were obtained using Agilent DSO6014 oscilloscope, Tektronix A6302 current probes, and then data were postprocessed by using MATLAB software (waveform plotting, reactive currents spectra, positive and negative-sequence calculations). The performed experiments used the same loads and the same controllers described in Sect. 3 and the setup of Fig. 10. Only steady state operation is shown, since the objective of the experimental results is mainly to validate the reference current calculation block.

Figure 18 shows the line to neutral voltages corresponding to the fundamental reactive current compensation using Load $1\left(S_{1}\right.$ and $S_{2}$ On) and Load $2\left(S_{3}\right.$ On).

Load currents, shown at the bottom of Fig. 18, presents high amplitude and are delayed from their corresponding line to neutral voltages. Compensated currents have lower amplitude and are visually in phase with the grid voltages.

Table 4 shows the fundamental symmetrical components of the grid voltage, the compensated grid current and the load current.

Reactive currents were compensated as expected (grid current amplitude is lower and its phase angle was reduced from $62.6^{\circ}$ to $-3.5^{\circ}$ ), and the results are similar to the ones obtained in Table 1 for the simulated case.

Figure 19 shows the fundamental negative-sequence compensation using Load $1\left(S_{1}\right.$ On and $S_{2}$ Off) for the simulated
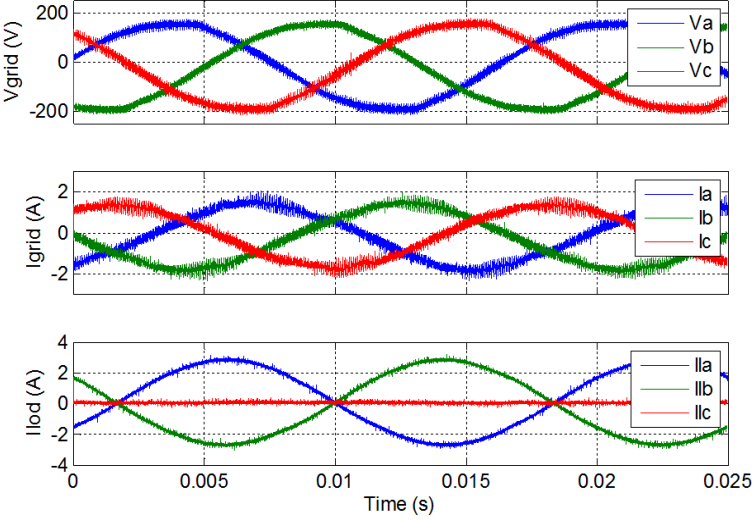

Fig. 19. Experimental results for fundamental negativesequence compensator waveforms: top - line to neutral voltages $\left(v_{a}, v_{b}, v_{c}\right)$, middle: line currents $\left(i_{a}, i_{b}, i_{c}\right)$, bottom- load currents $\left(i_{l a}, i_{l b}, i_{l c}\right)$

Table 5. Calculated symmetrical components for fundamental negative component compensation- Experimental results

\begin{tabular}{|c|c|c|c|}
\hline sequence & $\mathrm{V}_{\text {grid }}$ & $\mathrm{I}_{\text {grid }}$ & $\mathrm{I}_{\text {load }}$ \\
\hline positive & $175.82\left\lfloor 0.3^{\circ}\right.$ & $1.57-73.0^{\circ}$ & $1.59-76.8^{\circ}$ \\
\hline negative & $0.63\left[-80.4^{\circ}\right.$ & $0.03-3.1^{\circ}$ & $1.59 \mid-16.7^{\circ}$ \\
\hline zero & $0.29-137.8^{\circ}$ & $0.01-109.7^{\circ}$ & $0.02\left\lfloor-127.9^{\circ}\right.$ \\
\hline
\end{tabular}
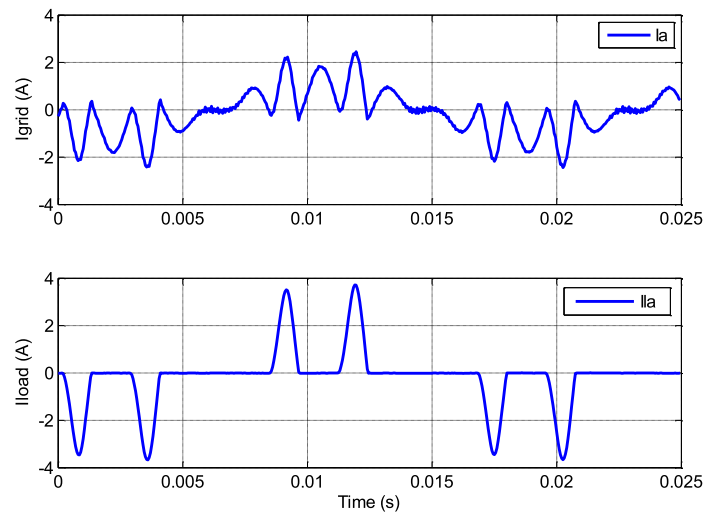

Fig. 20. Experimental results for current harmonics: top - line current $\left(i_{a}\right)$, bottom- load currents $\left(i_{l a}\right)$

case of Fig. 15. High amplitude load currents exist only at phases $a$ and $b$. Grid currents are balanced, with lower amplitude and reactive components, as expected. Table 5 summarizes the calculated symmetric components of the measured values, showing good agreement with the results presented in Table 2 for the simulated case. The negative-sequence was reduced from $1.59 \mathrm{~A}$ to $0.03 \mathrm{~A}$.

Figure 20 shows the compensation of $5^{\text {th }}$ and $7^{\text {th }}$ order harmonics using Load $2\left(\mathrm{~S}_{3}\right.$ on) for the simulated case of Fig. 15. Table 6 shows the calculated values of the $1^{\text {st }}, 5^{\text {th }}, 7^{\text {th }}, 11^{\text {th }}$ and $13^{\text {th }}$ harmonics amplitudes for phase a of grid and load currents.

Table 6 shows that the 5th and 7th harmonics were adequately compensated by the proposed method and that the results are similar to the simulated case shown in Table 3.

\section{Conclusion}

This paper has presented a method to individually calculate 
Table 6. Calculated $1^{\text {st }}, 5^{\text {th }}$ and $7^{\text {th }}$ current components (peak value A) for specific current harmonics compensation-Experimental results

\begin{tabular}{|c|c|c|}
\hline & $\mathrm{I}_{\text {grid }}($ peak value $\mathrm{A})$ & $\mathrm{I}_{\text {load }}($ peak value $\mathrm{A})$ \\
\hline 1 & 1.089 & 0.934 \\
\hline 5 & 0.036 & 0.841 \\
\hline 7 & 0.028 & 0.794 \\
\hline 11 & 0.563 & 0.569 \\
\hline 13 & 0.521 & 0.496 \\
\hline
\end{tabular}

the instantaneous positive, negative and zero-sequences of any current or voltage harmonic component, which is based on the dot product of three-dimensional space vectors in the stationary frame. The method can be applied to calculate the reference signals for voltage and current disturbance compensators. Simulated data have shown the compensation of reactive power, load unbalance and current harmonics, and all these three cases were experimentally verified, validating the algorithm. The proposed method is similar to the modified $p q$ theory method. Both algorithms present different approaches for explaining the same phenomena. Moreover, the proposed and the $d q$ methods require twelve multiplications and evaluation of six trigonometric functions. Therefore, they have the same computational effort.

Considering the complete converter control system, the advantages of this approach over the $d q$ implementation are:

- All measurements are performed in the original $a b c$ system. In $d q$ frame control, all measurement must be transformed to synchronous frame, increasing computational effort;

- Since the actuation must occur in the original $a b c$ reference frame, computational effort is also decreased;

- All the control loops are performed in $a b c$ coordinates, as it is commonly found in literature ${ }^{(11)(13)-(15)}$ and $^{(16)}$.

\section{Acknowledgment}

The authors are grateful to Texas Instruments Academic Program, for donating the DSP Evaluation Kit and respective software, and to Council for Scientific and Technological Development (CNPq), São Paulo Research Foundation (FAPESP- grant 2014/03700-6) and FDTE for the financial support.

\section{References}

( 1 ) H. Akagi, Y. Kanazawa, and A. Nabae: "Instantaneous Reactive Power Compensators Comprising Switching Devices without Energy Storage Components", IEEE Trans. Industry Applications, Vol.IA-20, No.3, pp.625, 630 (1984)

( 2 ) H. Akagi, E.H. Watanabe, and M. Aredes: "Instantaneous Power Theory and Applications to Power Conditioning”, John Wiley and Sons, p.380 (2007)

( 3 ) P. Tenti, H.K.M. Paredes, and P. Mattavelli: "Conservative Power Theory, a Framework to Approach Control and Accountability Issues in Smart Microgrids", IEEE Trans. on Power Electronics, Vol.26, No.3, pp.664-673 (2011)

( 4 ) T. Xiangqian, S. Ming, Y. Jun, and L. Mu: "Single order harmonic detection with incremental algorithm of DFT", Power Electronics and Motion Control Conference, 2009. IPEMC '09. IEEE 6th International, Vol.1, No.1, pp.2402-2405 (2009)

( 5 ) X. Jin, H. Min, C. Haiyun, and F. Wei: "A Detection Approach for Random Harmonic Current in Single-Phase Circuit", Intelligent System Design and Engineering Application (ISDEA), 2012 Second International Conference on, Vol.1, No.1, pp.1346-1350 (2012)

(6) L. Asiminoaei, F. Blaabjerg, and S. Hansen: "Detection is key-Harmonic detection methods for active power filter applications", Industry Applications Magazine, IEEE, Vol.13, No.4, pp.22-33 (2007)
( 7 ) S. Shiping, L. Guiying, L. Jianing, and Q. Zhiqing: "A Specific Harmonic Detection Method for Three-Phase Four-Wire Circuits Based on Multifrequency Rotating Coordinate Transformation", Electrical and Control Engineering (ICECE), 2010 International Conference on, Vol.1, No.1, pp.261264 (2010)

( 8 ) R. Cutri and L. Matakas: "A Generalized Instantaneous Method for Harmonics, Positive and Negative Sequence Detection/Extraction", Power Electronics Specialists Conference, 2007. PESC 2007. IEEE, Vol.1, No.1, pp.2294, 2297 (2007)

( 9 ) P. Tenti, J. Willems, P. Mattavelli, and E. Tedeschi: "Generalized Symmetrical Components for Periodic Non-sinusoidal Three-phase Signals", Electrical Power Quality and Utilisation Journal, Vol.XIII, No.1, pp.9-15.

(10) M.S. Padua, S.M. Deckmann, and F.P. Marafao: "Frequency-Adjustable Positive Sequence Detector for Power Conditioning Applications", Power Electronics Specialists Conference, 2005. PESC '05. IEEE 36th, Vol.1, No.1, pp.1928-1934 (2005)

(11) S. Buso and P. Mattavelli: Digital Control in Power Electronics. 1st ed. San Rafael: Morgan \& Claypool, p.151 (2006)

(12) D.G. Holmes and T.A. Lipo: "Pulse width modulation for power converters: principles and practice”, IEEE Press Series Power Eng. (2003)

(13) L. Matakas, W. Komatsu, and F.O. Martinz: "Positive sequence tracking Phase Locked Loops: A unified graphical explanation", Power Electronics Conference (IPEC), 2010 International, Vol.1, pp.1273-1280 (2010)

(14) N.R.N. Ama, W. Komatsu, F. Kassab Junior, and L. Matakas Junior: "Adaptive single phase moving average filter PLLs: analysis, design, performance evaluation and comparison", Przeglad Elektrotechniczny, No.3 (2014)

(15) A.G. Yepes, F.D. Freijedo, J. Doval-Gandoy, O. Lopez, J. Malvar, and P. Fernandez-Comesana: "Effects of Discretization Methods on the Performance of Resonant Controllers", IEEE Transactions on Power Electronics, Vol.25, No.7, pp.1692-1712 (2010)

(16) D.G. Holmes, T.A. Lipo, B.P. McGrath, and W.Y. Kong: "Optimized Design of Stationary Frame Three Phase AC Current Regulators", IEEE Transactions on Power Electronics, Vol.24, No.11, pp.2417-2426 (2009)

\section{Appendix}

PLL

The implemented PLL block is shown in app. Fig. 1 .

The Voltage Controlled Oscillator (VCO) block generates two sets of unitary amplitude, positive-sequence signals, with frequency $\omega_{P L L}$, that are the coordinates of vectors $\vec{V}_{P L L 1 \perp}^{+}$ and $\vec{V}_{P L L 1 \|}^{+}$. The phase detector block calculates the dot product between $\vec{V}$ and $\vec{V}_{P L L 1 \perp}^{+}$, and attenuates the oscillating signals with zero mean value by means of a low pass filter. The PI controller changes $\omega_{P L L}$ in a way to force $\vec{V}_{P L L 1 \perp}^{+}$to be in quadrature with the fundamental positive-sequence of $\vec{V}$, and $\vec{V}_{P L L 1 \|}^{+}$to be in phase with the fundamental positive-sequence of $\vec{V}$. The PI controller is adjusted according to ${ }^{(14)}$. This paper uses a moving average filter with $T_{W} / 2$ window as a low pass filter.

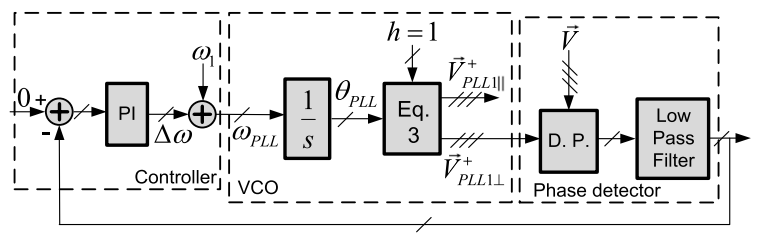

app. Fig. 1. PLL block diagram 
Kelly Caroline Mingorancia de Carvalho (Non-member) received

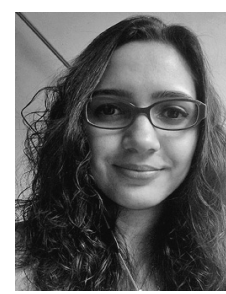
the B.S. and Master degrees in electrical engineering from the Polytechnic School of the University of São Paulo, São Paulo, Brazil, in 2012 and 2015 respectively. Currently, she is pursuing the Ph.D. degree in electrical engineering at the Polytechnic School of the University of São Paulo. Her current research interests include power quality, active power filters, modular multilevel converters and disturbances detection in power systems.

Naji Rajai Nasri Ama (Non-member) received the B.S. and the M.S

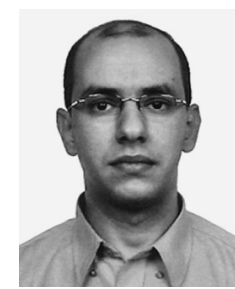
degrees in electrical engineering from the University of Technology, Baghdad, Iraq, in 2000 and 2003, respectively and Ph.D. degrees from the Polytechnic School of the University of São Paulo (EPUSP), São Paulo, Brazil, in 2012. Currently, he is an Associate Professor at Dom Bosco Catholic University (UCDB). His research interests include grid synchronization methods, power electronics, digital signal processing, control systems, and grid-connected

power converters.

Wilson Komatsu (Non-member) was born in São Paulo, Brazil, in 1963. He received the B.S., M.S., and Ph.D. degrees in electrical engineering from the Escola Politécnica da Universidade de São Paulo (EPUSP), São Paulo, in 1986, 1992, and 2000, respectively. He is Associate Professor of power electronics at EPUSP. His research areas are control and modeling of static converters and their application to electrical power systems and electrical power quality. Dr. Komatsu is a member of the Institute of Electrical and Electronics Engineers (IEEE) and of the Brazilian Power Electronics Society (SOBRAEP).
Fernando Ortiz Martinz (Non-member) received the B.S. degree in

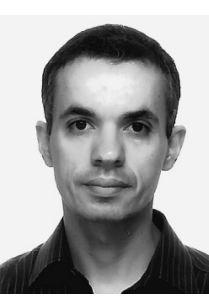
electrical engineering from the State University of Campinas, São Paulo, Brazil, in 2003, and the M.S. and Ph.D. degrees from the Polytechnic School of the University of São Paulo (EPUSP), São Paulo, Brazil, in 2007 and 2013, respectively. Currently, he works as a researcher at EPUSP. His interests include modeling, control, and synchronization in power electronics, power quality, and grid-connected power converters. Mr. Martinz is a member of the Brazilian Power Electronics Society (SOBRAEP).

Ricardo Souza Figueredo (Non-member) received the B.S. degree

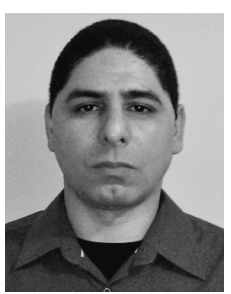
in electrical engineering from the Pontifical Catholic University of Sao Paulo, São Paulo, Brazil, in 2010. and the M.S. degree in electrical engineering from the Polytechnic School of the University of São Paulo, São Paulo, Brazil, in 2015. His current research interests include design and modelling of power converters for renewable energy sources and digital control of power converters.

Lourenço Matakas Jr. (Non-member) received the B.S., M.S., and

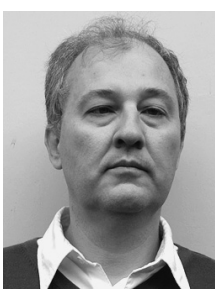

$\mathrm{Ph} . \mathrm{D}$. degrees in electrical engineering from the Escola Politecnica da Universidade de Sao Paulo, Sao Paulo, Brazil, in 1984, 1989, and 1998, respectively, where he is an Associate Professor since 1996. His research focus is on topologies, control and application of static converters connected to the grid, phaselocked loops, and pulsewidth modulation strategies. $\mathrm{He}$ is a member of the Brazilian Power Electronics Society (SOBRAEP) and IEEE. 